\title{
Improved computer-assisted analysis of the global lymphatic network in human cervical tissues
}

\author{
Cédric Balsat ${ }^{1,7}$, Nicolas Signolle ${ }^{1,7}$, Frédéric Goffin ${ }^{2}$, Katty Delbecque ${ }^{3}$, \\ Benoit Plancoulaine ${ }^{4}$, Philippe Sauthier ${ }^{5}$, Vanessa Samouëlian ${ }^{5}$, Aude Béliard ${ }^{2}$, \\ Carine Munaut ${ }^{1}$, Jean-Michel Foidart ${ }^{1}$, Silvia Blacher ${ }^{1}$, Agnès Noël ${ }^{1}$ and Frédéric Kridelka ${ }^{6}$ \\ ${ }^{1}$ Laboratory of Tumor and Development Biology, Groupe Interdisciplinaire de Génoprotéomique Appliquée \\ (GIGA-Cancer), University of Liège, Pathology Tower (B23), Liège, Belgium; ${ }^{2}$ Department of Obstetrics and \\ Gynecology, Hospital of la Citadelle, Liège, Belgium; ${ }^{3}$ Department of Pathology, Hospital of la Citadelle, \\ Liège, Belgium; ${ }^{4}$ GRECAN, University of Caen, François Baclesse Comprehensive Cancer Center, Caen, \\ France; ${ }^{5}$ Departement of Gynecologic Oncology, CHU of Montréal, Montréal, Canada and ${ }^{6}$ Department of \\ Obstetrics and Gynecology, CHU of Liège, Liège, Belgium
}

\begin{abstract}
Lymphatic dissemination is a key event in cervical cancer progression and related tumor lymphatic markers are viewed as promising prognostic factor of nodal extension. However, validating such parameters requires an objective characterization of the lymphatic vasculature. Here, we performed a global analysis of the lymphatic network using a new computerized method applied on whole uterine cervical digital images. Sixty-eight cases of cervical neoplasia (12 CIN3, 10 FIGO stage $1 \mathrm{~A}$ and 46 stage IB1) and 10 cases of normal cervical tissue were reacted with antibodies raised against D2-40, D2-40/p16 and D2-40/Ki67. Immunostained structures were automatically detected on whole slides. The lymphatic vessel density (D2-40), proliferating lymphatic vessel density (D2-40/ki67) and spatial lymphatic distribution in respect to the adjacent epithelium were assessed from normal cervix to early cervical cancer and correlated with lymphovascular space invasion and lymph node status. Prominent lymphatic vessel density and proliferating lymphatic vessel density are detected under the transformation zone of benign cervix and no further increase is noted during cancer progression. Notably, a shift of lymphatic vessel distribution toward the neoplastic edges is detected. In IB1 cervical cancer, although intra- and peritumoral lymphatic vessel density are neither correlated with lymphovascular space invasion nor with lymph node metastasis, a specific spatial distribution with more lymphatic vessels in the vicinity of tumor edges is predictive of lymphatic dissemination. Herein, we provide a new computerized method suitable for an innovative detailed analysis of the lymphatic network. We show that the transformation zone of the benign cervix acts as a baseline lymphangiogenic niche before the initiation of neoplastic process. During cancer progression, this specific microenvironment is maintained with lymphatic vessels even in closer vicinity to tumor cells.
\end{abstract}

Modern Pathology (2014) 27, 887-898; doi:10.1038/modpathol.2013.195; published online 6 December 2013

Keywords: cancer; cervix; computerized; distribution; lymphatic; metastasis

In uterine cervical neoplasms, and in most human solid tumors, lymphatic vasculature is the common pathway for initial tumor spread to regional lymph nodes. ${ }^{1}$ In addition to tumor size, lymph node status (positive/negative lymph node metastasis) is

Correspondence: Professor F Kridelka, PhD, CHU of Liège, Service de Gynécologie-Obstétrique, 600 Rue Gaillarmont 4030, Grivegnée, Belgium.

E-mail: frederic.kridelka@chu.ulg.ac.be

${ }^{7}$ These authors contributed equally to this work.

Received 13 May 2013; revised 20 September 2013; accepted 23

September 2013; published online 6 December 2013 regarded as the strongest prognostic factor for patient's cancer-specific survival. ${ }^{2,3}$ It is widely used for guiding therapeutic decisions and currently justifies a systematic lymphadenectomy in early cervical cancer. Owing to the low rate of positive lymph node detected (ranging from 12 to $22 \%)^{4}$ and the potential morbidity associated with a lymph node dissection, research has focused over recent years on the identification of primary tumor variables predictive of lymphatic spread. ${ }^{5,6}$

In this context, lymphovascular space invasion has been described as an independent factor predicting tumor aggressiveness. ${ }^{7}$ More recently, the 
lymphatic vessel density, which witnesses the level of lymphangiogenesis (formation of new lymphatic vessels), has been proposed as a new promising predictive variable of nodal extension. ${ }^{8}$ Notably, studies on cervical tissues showed that high levels of lymphatic vessel density as well as marked expression of vascular endothelial growth factors (VEGF)-C and VEGF-D, the two main lymphangiogenic promoters, are already detected in preinvasive neoplasia (CIN3). ${ }^{9-12}$ A gradual increase of lymphatic vessel density has been reported in the peritumoral stroma during cancer progression ${ }^{13-15}$ and in invasive cervical cancer, Gombos et $a l^{15}$ were the first to link high peritumoral lymphatic vessel density with growth factor expression, lymphovascular space invasion, lymph node metastases and overall survival. Subsequent immunohistochemical studies on cervical neoplasms confirmed a positive association between lymphatic vessel density and lymph node metastases. ${ }^{14,16-21}$ Taken together, these results suggest an early lymphangiogenesis switch with an increased risk of lymphatic dissemination during cancer progression that could be assessed by lymphatic vessel density quantification. However, until recently, lymphatic vessel density quantification has relied on the hot spot technique previously criticized for its subjectivity. The main disadvantages of this approach are its focus on a limited tumor area and its lack of reproducibility. ${ }^{22}$ Consequently, no consensus exists on the potential prognostic value of the tumor lymphatic vasculature profile in cervical cancer. ${ }^{10,23,24}$

In order to overcome the major restrictions related to the conventional hot spot approach, we have developed an original semi-automated quantification method based on computerized image analysis of lymphatic vessels on whole slide scanned tissue sections. ${ }^{25}$ The present work aims at drawing pathologist's attention on the interest of a global lymphatic vasculature analysis on cancer tissues. In particular, we provide evidence for a so far unappreciated lymphatic niche under the transformation zone considered as the site where neoplastic changes occur primarily during the cervical oncogenic process. ${ }^{26,27}$ Furthermore, our study demonstrates that a global stromal evaluation sheds light on topographical features of the lymphatic vascular network that are worth considering in cervical cancer as well as other cancers.

\section{Materials and methods}

\section{Tissue Samples}

Surgical specimens of 68 patients suffering cervical neoplasia were obtained from the biobanks of the University of Liège (CHU, Liège Belgium) and the Centre Hospitalier Universitaire of Montreal (CHUM, Hôpital Notre-Dame, Montreal Canada) after study approval by local ethic committees.
Twelve cases of in situ carcinomas (CIN3: presence of neoplastic cells that occupy the full thickness of the epithelium), 10 microinvasive lesions (FIGO stage IA1: neoplastic process $\leq 3 \mathrm{~mm}$ of depth and $\leq 7 \mathrm{~mm}$ of width) and 46 early uterine cervical cancers (IB1: clinically visible lesion $\leq 4 \mathrm{~cm}$ in greatest axis) were successively collected. Stage was allocated according to the International Federation of Gynecology and Obstetrics staging system (FIGO). Lymph node metastases were detected in $12 / 46$ invasive cases and lymphovascular space invasion was present in 25/46 surgical specimens. Ten benign uterine cervical samples were also obtained from patients treated for non-oncological reasons (fibroma, menorrhagia, uterine prolapse, Lynch syndrome). The cervix was sectioned and sections were oriented according to standard histological guidelines. ${ }^{28}$ All tissues were reviewed by a pathologist blinded to the study purpose. CIN3 and microinvasive lesions were diagnosed on a cone biopsy specimen that encompasses a large part of the tree anatomical regions of the uterine cervix. Early cervical cancer lesions and benign cervix samples of $0.5 \mathrm{~cm}$ thickness were collected after radical surgical hysterectomy. For early cervical cancers, this sampling was performed in the most infiltrative area. Among representative slides, those permitting optimal transformation zone (normal cervix), dyspalsia or micro-invasive cancers evaluation as well as peritumoral stromal immunostaining were selected.

\section{Immunohistochemisty}

Lymphatic vessels, proliferating lymphatic vessels and tumor cells were identified using a monoclonal anti-human antibody against podoplanin (D2-40, 1:100; Dako, Heverlee, Belgium), a prediluted multiplex cocktail composed of monoclonal mouse anti D2-40 and rabbit monoclonal anti-Ki67 (ready to use; Biocare Medial, Concord, USA) and a polyclonal rabbit anti CDKN2A/p16 ${ }^{\text {INK4a }}$ (1:100; Abcam, Cambridge, UK), respectively.

\section{Virtual Image Acquisition and Processing}

Virtual images were acquired with the fully automated digital microscopy system dotSlide (Olympus, BX51TF, Aartselaar, Belgium). Whole tissue sections were digitized at high magnification producing virtual images in which pixels measure $0.4225 \mu \mathrm{m}^{2}$. DotSlide providing images in a proprietary format, images were converted into a standard TIFF format, easier to handle. Thereafter, tumor cells and lymphatic vessels were computerized as illustrated in Figure 1. When Ki67-positive lymphatic vessel sections were considered, because of low contrast between podoplanin and Ki67-positive nuclei, vessels were manually drawn with the help of the Aperio ImageScope v10.2.1.2314 software. 

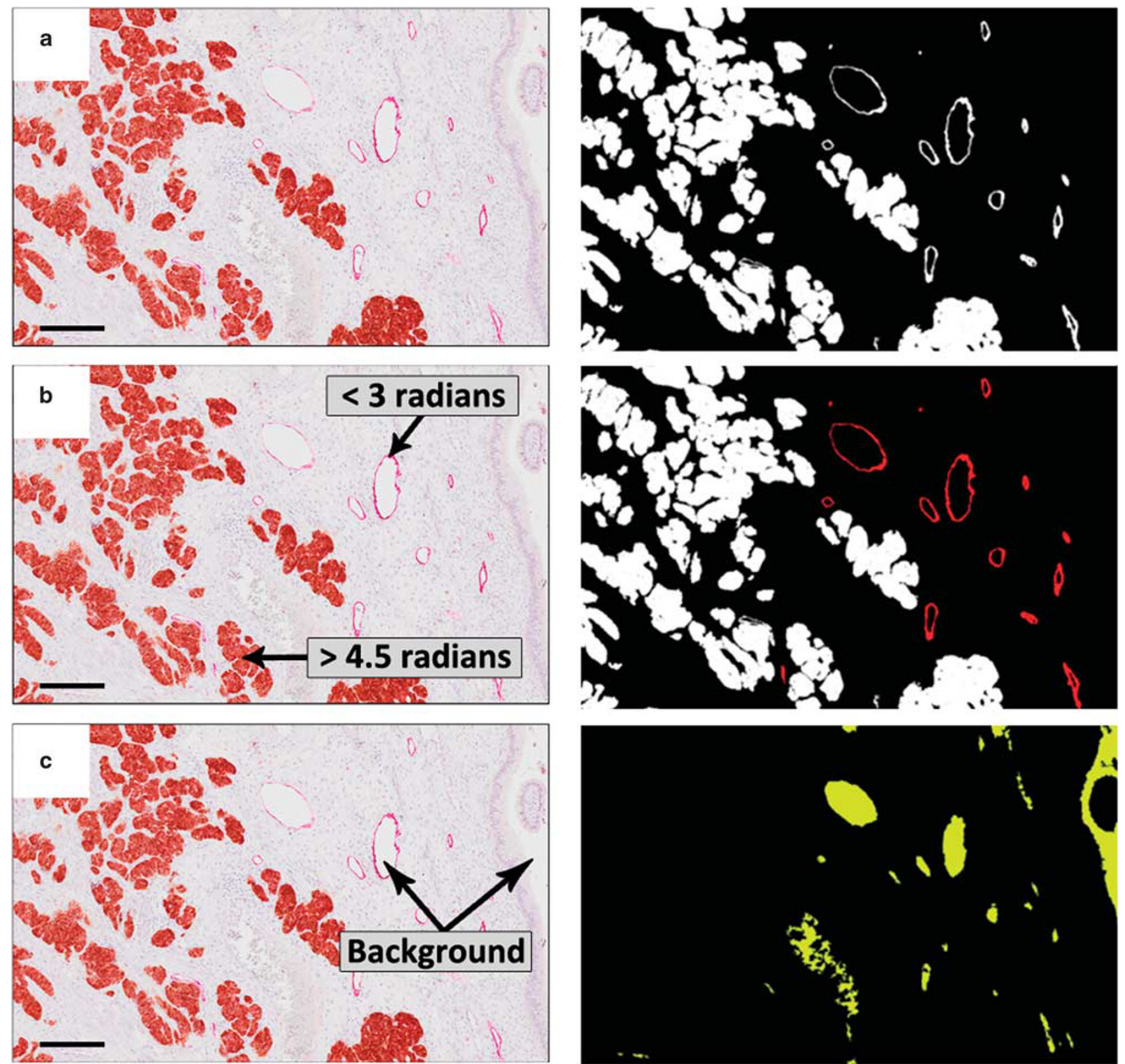

Figure 1 Illustration of tumor cell and lymphatic vessel detection. Virtual images of immunostained cervical cancer tissues are presented on the left and corresponding binary images are shown on the right. (a) Tumor cells (brown, left image) and lymphatic vessels (red, left image) are automatically segmented together thanks to a Maximum Entropy Thresholding performed on the excess red component. (b) They are automatically distinguished based on the average value of the color presented by each detected structure $(>4.5$ radians $=$ tumor cell in white, $<3$ radians = lymphatic vessel in red). (c, d) In order to take into consideration lymphatic vessels in their integrality, vessel lumens are filled. Background is firstly segmented (c, yellow) and detected structures in close contact with lymphatic wall are considered (d). (e) Finally, to avoid misinterpretations, non-vascular structures that could have been detected and/or detection mistakes are manually corrected (green arrows, right image). (f) The final binary image of detected tumor cells and lymphatic vessels (right image) is obtained after the image processing. Scale bars represent $200 \mu \mathrm{m}$.

The three anatomical regions (exocervix, transformation zone and endocervix) were delineated on virtual images by using the Aperio ImageScope v10.2.1.2314 software. The transformation zone was defined as the cervical area in which squamous and columnar epithelia were present in continuity and/or squamous epithelium was detected with underlying glands. ${ }^{29}$ Epithelia of benign cervical tissues, CIN3 and microinvasive lesions as well as the boundary between intra- and peritumoral areas in FIGO 1B1 cervical cancers were delimited in the same way. Finally, tissues were extracted by a moment-preserving thresholding method ${ }^{30}$ applied on the blue component of the original virtual image. 

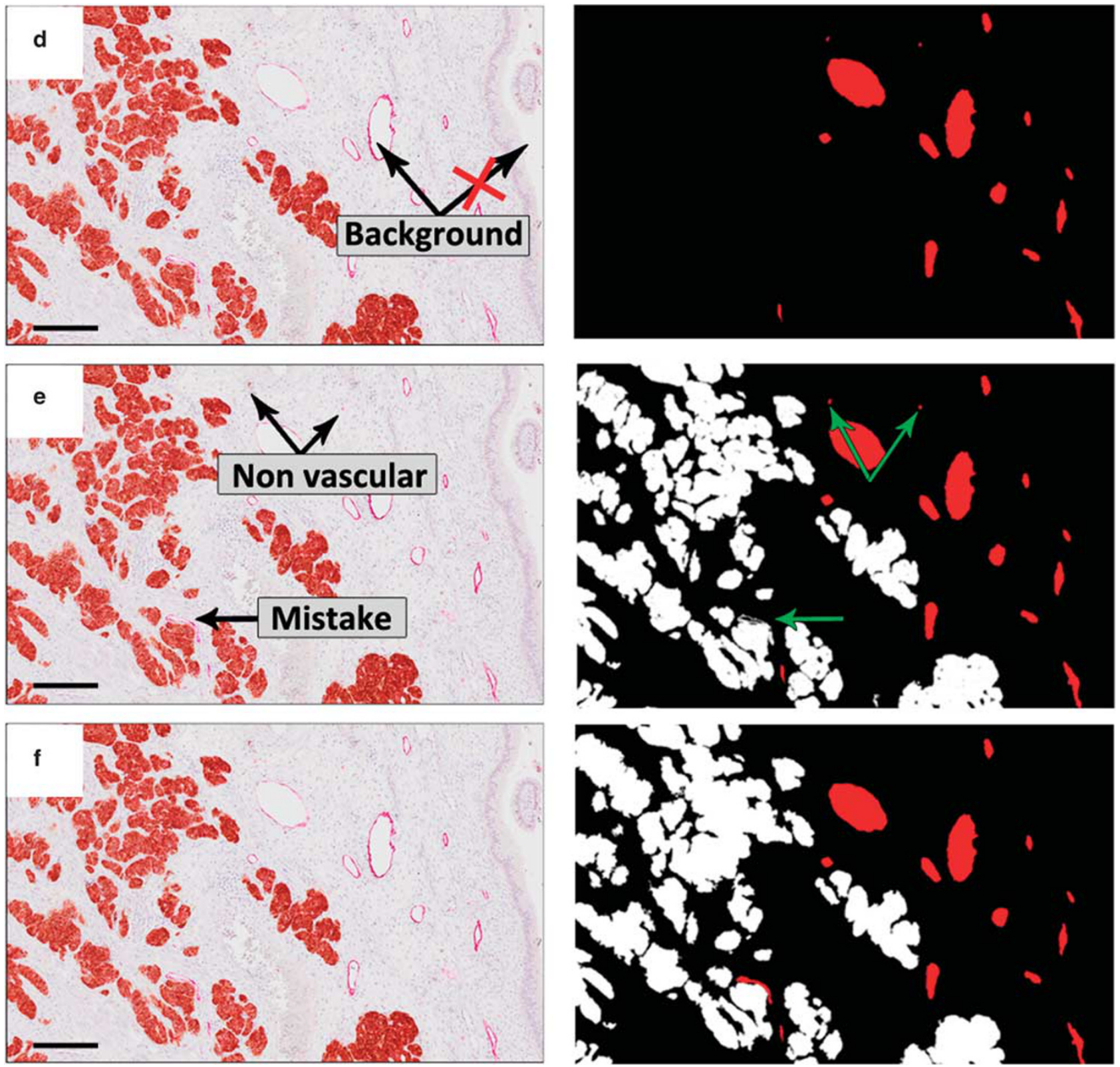

Figure 1 (Continued).

Owing to high image size that hampers calculation, all binary images of the detected structures were decimated according to the procedure described elsewhere. ${ }^{31}$ Figure 2 illustrates the different steps of image processing.

\section{Image Analysis}

Image analysis and measurements were performed using image analysis library Pandore (GREYC, Caen, France), Aphelion 3.2 (ADCIS, Saint-Contest, France) and image toolbox of MATLAB 9.2 (MathWorks, Natick, MA, USA) software. Lymphatic vessel density, proliferating lymphatic vessel density and spatial distribution of lymphatic vessel were quantified from

the basal layer of the surface epithelium or neoplastic edges to tissue border. Lymphatic vessel density and proliferating lymphatic vessel density were defined as the number of lymphatic vessels and Ki67-positive lymphatic vessels per $\mathrm{mm}^{2}$ of tissue, respectively. Spatial lymphatic vessel distribution analysis was performed by measuring the Euclidean distance between the center of mass of each vessel section and the basal layer of the surface epithelium or neoplastic edges as previously described. ${ }^{25}$ For comparison, curves were normalized taking the maximum of the control curve at one.

In CIN3, microinvasive and IB1 affected tissues, the perineoplastic area was defined as the stromal region located from neoplastic edges to $2 \mathrm{~mm}$ of depth. The transformation zone of benign cervix was 
a

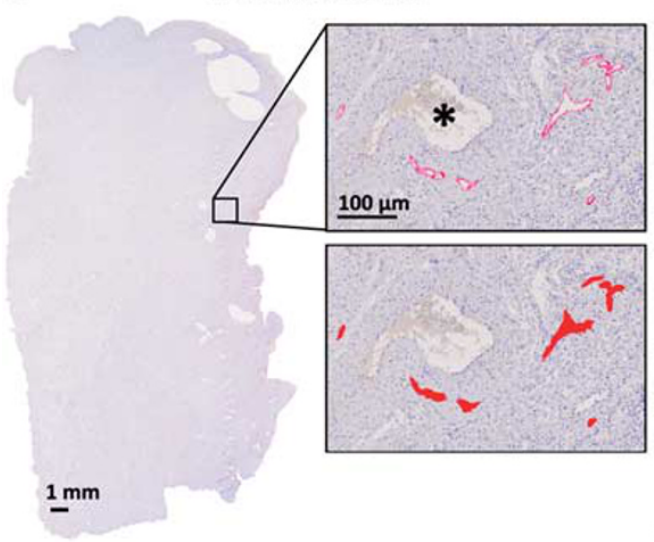

e b

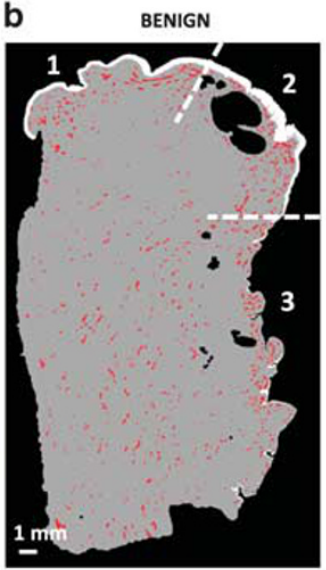

f

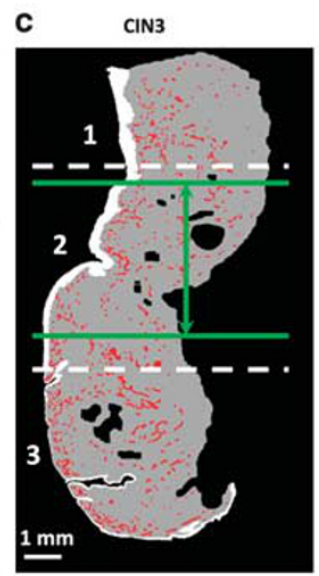

INVASIVE

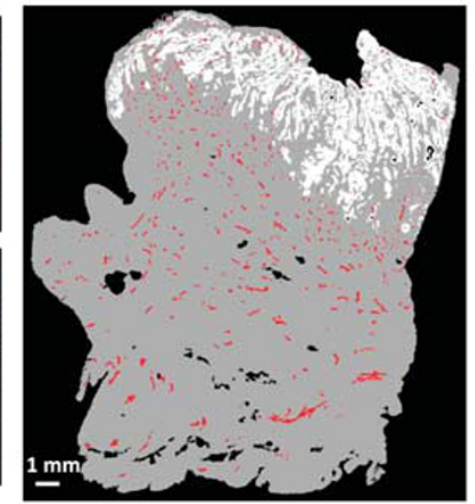

g d MICROINVASION

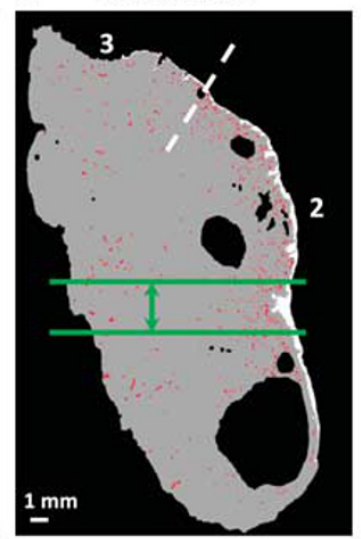

INVASIVE
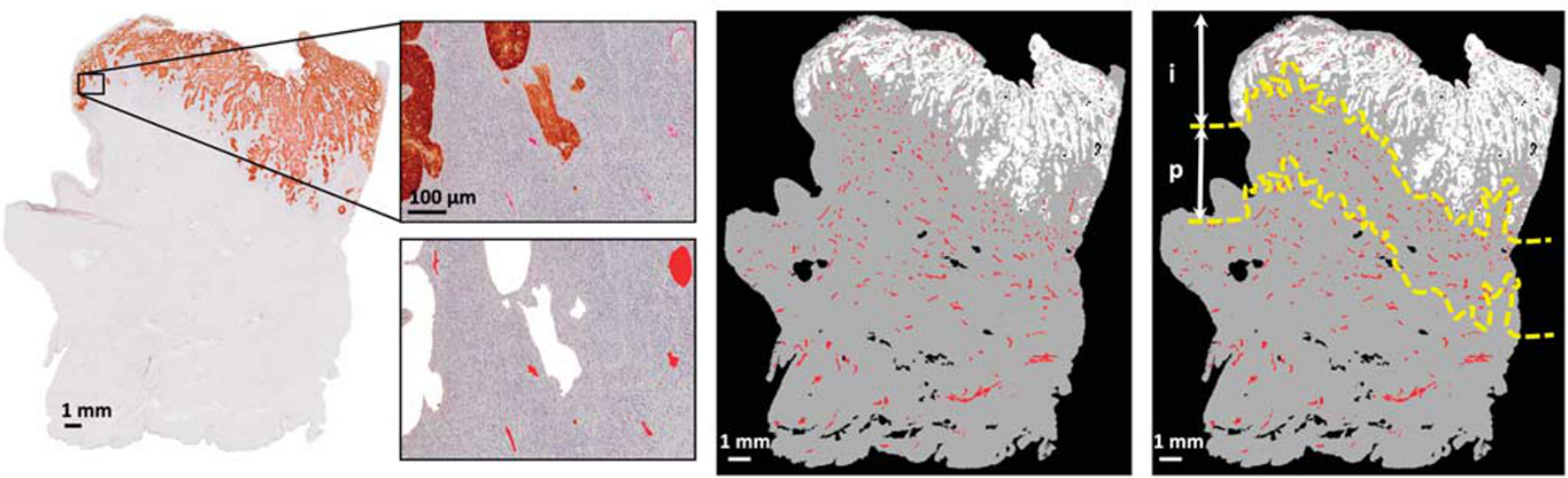

Figure 2 Image processing illustration. (a) Virtual image of whole benign cervical tissue. Lymphatic vessel sections were detected by immunostaining with the anti-podoplanin antibody (D2-40) and visualized in pink as illustrated at higher magnification in the insert (top). Lymphatic vessel section edges and lumens were automatically segmented on virtual image (red) while blood vessels were not detected (star). (b-d) Binary images of detected lymphatic vessel sections (red), tissue (grey) and epithelium (white) were generated. Exocervix (1), transformation zone (2) and endocervix (3) were manually delineated (white dotted lines). Neoplastic lesions and their underlying stromal tissues were manually highlighted in CIN3 (c) and microinvasive lesions (d) (green lines). Green arrow represents tissue under neoplastic lesion that was analyzed. (e) Virtual image of whole cervical cancer slide. In invasive lesions, lymphatic vessel sections (pink) and tumor cells (brown) were detected by immunostaining with D2-40 and CDK2A/P16 ${ }^{\text {IN4Ka }}$ antibodies, respectively. High magnification is shown in the insert. Lymphatic vessel sections (red) and tumor nodules (white) were automatically and discriminatory segmented (bottom insert). (f) Binary image of lymphatic vessel sections and tumor nodules on the whole slide (g). Intratumoral (i) and peritumoral regions (p) were manually discriminated (yellow dotted line). Peritumoral area was considered as the area located within $2 \mathrm{~mm}$ of depth from the tumor edges. White arrows represent the intra- and peritumoral areas that were analyzed.

considered as reference to which quantifications obtained under the neoplastic lesions were compared.

\section{Statistical Analysis and Graphs}

For lymphatic vessel density, proliferating lymphatic vessel density and vessel distance to epithelium/ tumor border, data were analyzed with GraphPad Prism 5.0 software (San Diego, CA, USA). Mean values were each compared using the MannWhitney test to determine whether a difference between experimental groups could be considered as significant. Statistical analysis of lymphatic vessel spatial distribution was performed using a Wilcoxon non-parametric test with the statistic toolbox of MATLAB 9.2 software. The level of statistical significance was set at $P<0.05$ for all comparisons. Graphs are presented as scatter plots of individual data points. Means are presented by horizontal bars.

\section{Results}

\section{Hot Spot versus Computer-Assisted Analysis}

Intra- and peritumoral lymphatic vessel density were first assessed using the hot spot technique by two independent operators according to the method described by Weidner et $a l^{32}$ and then compared with that determined by a computerized method on 46 cases of early cervical cancers. Inter-observer variability obtained after quantifications within the hot spot regions reaches $33.8 \%$ and $23.6 \%$ for 
intra- and peritumoral lymphatic vessel density, respectively. Also intra-observer variation exceeds $40 \%$ for both intra- and peritumoral lymphatic vessel density. Using the computer-assisted technique, we first determined that $93 \pm 3.5 \%$ of the lymphatic vessels can be accurately detected on whole tissue sections. Moreover, the quantification of intra- and peritumoral lymphatic vessel density on five successive slides reveals that the variability does not exceed $5 \%$.

\section{Lymphatic Vessel Density}

When analyzing the lymphatic vasculature of the benign cervical stroma, we observe a prominent lymphatic vessel density from the basal layer of the epithelium to a depth of $2 \mathrm{~mm}$. Beyond this depth, lymphatic vessels are sparsely distributed and show a significant and acute drop in density $(P<0.0001$; Figures 3a and b). Based on this observation, the lymphatic vasculature has been characterized thereafter in the stromal layer located within $2 \mathrm{~mm}$ of the adjacent subepithelial basal membrane or the nearest neoplastic invasive tumor edge. When considering specifically the stroma of exocervical, endocervical and transformation zone regions of the normal cervix (Figures 3c-e), a high lymphatic vessel density is detected under the transformation zone. In the transformation zone and exocervix, lymphatic vessels are distributed homogeneously

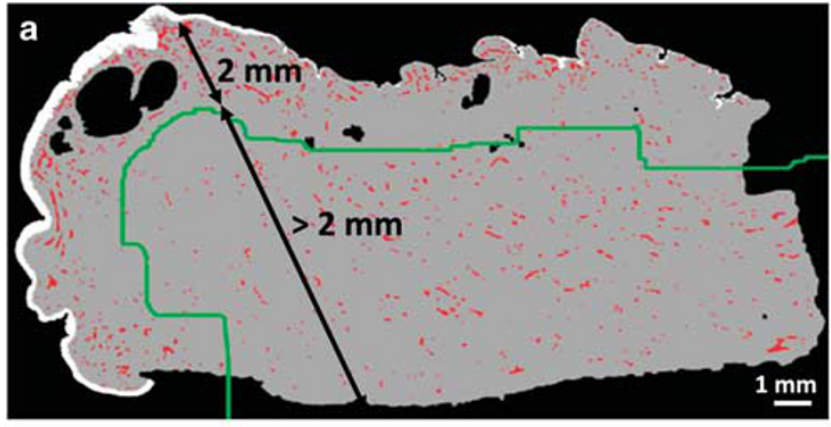

b
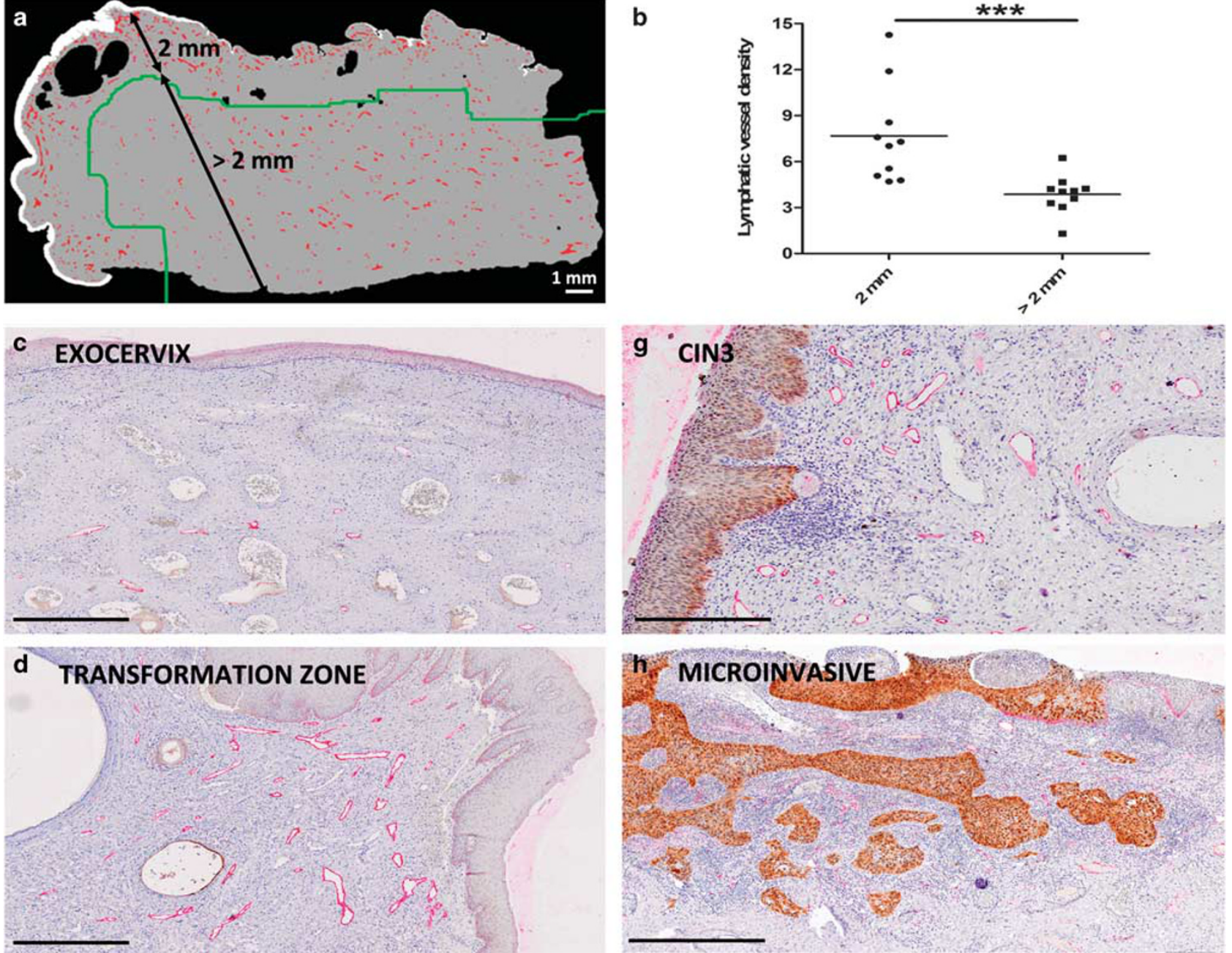

Figure 3 (a) Binary image of benign cervical tissue section. The green line represents the bottom limit of the stromal layer located within $2 \mathrm{~mm}$ of depth from the epithelium basis. (b) Lymphatic vessel density measured within $2 \mathrm{~mm}$ of depth in comparison with that detected beyond this limit in benign cervical tissue. (c-e) Lymphatic vessel immunostaining (D2-40, pink) within the exocervix, the transformation zone and the endocervix in benign cervical tissue. For the endocervix, pure glandular epithelium (left) and immature squamous metaplastic zone (right) were presented. (f) Lymphatic vessel density measured under the three anatomical regions of the benign uterine cervix (Endo, endocervix; Exo, exocervix; TZ, transformation zone). (g-i) Lymphatic vessel (D2-40, pink) and neoplastic cells (p16 ${ }^{\text {INK4a }}$, brown) immunostaining for (g) CIN3, (h) microinvasive and (i) 1B1 cervical neoplasia. (j) Lymphatic vessel density measured within the perineoplastic area of CIN3, microinvasive (MI) and 1B1 cervical lesions in comparison with non-pathological condition represented by the transformation zone of benign cervix (BC_TZ). Lymphatic vessel densities are expressed as the number of lymphatic vessel sections $/ \mathrm{mm}^{2}$. Scale bars represent $500 \mu \mathrm{m} .{ }^{*} P<0.05,{ }^{* *} P<0.001$. 

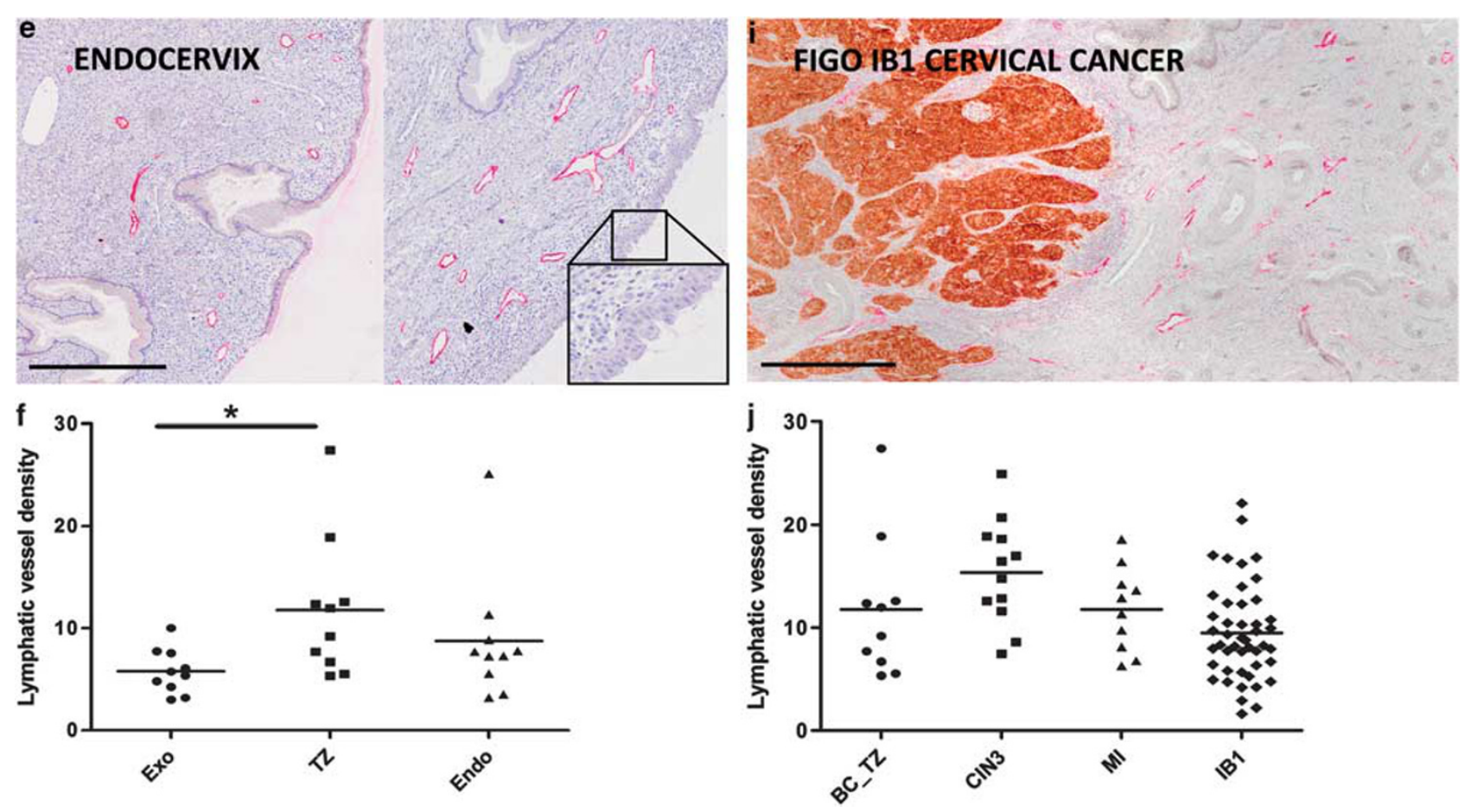

Figure 3 (Continued).
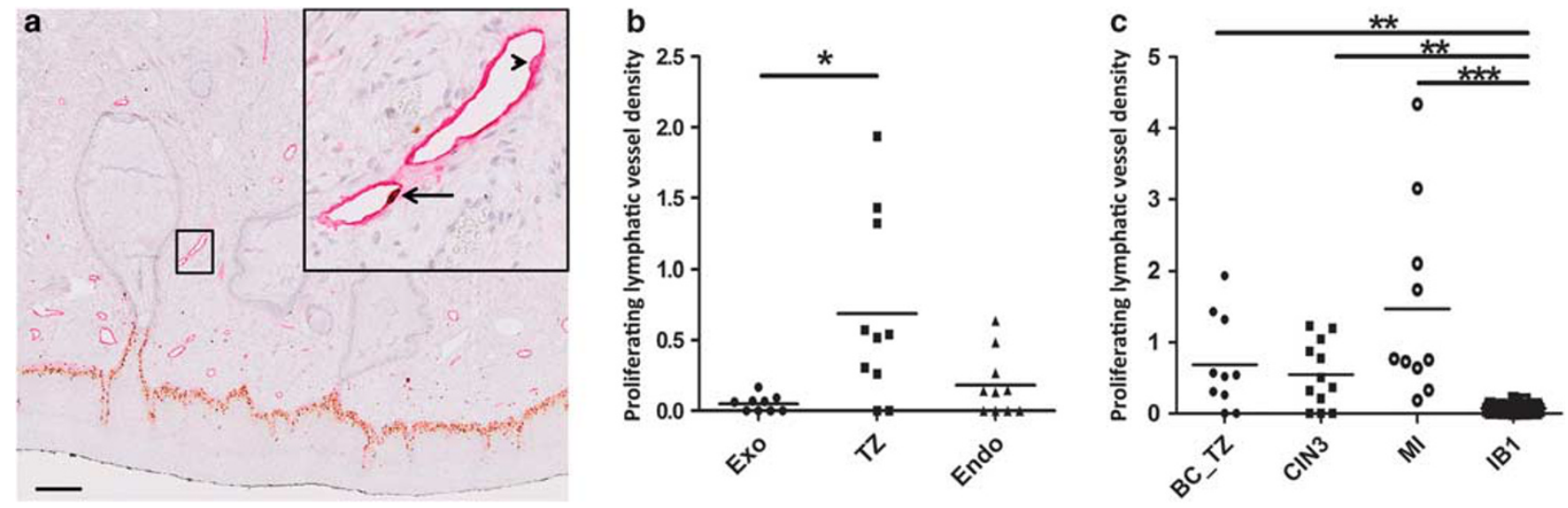

Figure 4 (a) Double immunostaining of lymphatic endothelial cells (D2-40, pink) and proliferating cells (Ki67, brown). Magnification represents a proliferating lymphatic vessel with Ki67-positive endothelial cell (arrow) next to a section with quiescent endothelial cell (arrow head). Scale bar represents $500 \mu \mathrm{m}$. (b) Proliferating lymphatic vessel density measured under the three anatomical regions of the benign uterine cervix (Endo, endocervix; Exo, exocervix; TZ, transformation zone). (c) Proliferating lymphatic vessel density measured within the perineoplastic area of CIN3, microinvasive (MI) and 1B1 cervical lesions in comparison with non-pathological condition represented by the transformation zone of benign cervix (BC_TZ). Proliferating lymphatic vessel density is expressed as the number of lymphatic vessel sections $/ \mathrm{mm}^{2} .{ }^{*} P<0.05,{ }^{* *} P>0.01,{ }^{* * *} P<0.001$.

while at the level of endocervix, they are heterogeneously distributed with low lymphatic vessel density under pure glandular epithelium and high lymphatic vessel density under immature squamous metaplastic zones that may be regarded as a focal transformation zone effect. The transformation zoneassociated lymphatic vessel density is higher than lymphatic vessel density observed under the exocervix $(P=0.0115)$ and not statistically different from that measured under the endocervix $(P=0.2475$; Figure $3 f)$.

Given the importance of the transformation zone in cervical oncogenesis, ${ }^{26,27}$ we next compared lymphatic vessel density under the transformation zone of benign cervix, CIN3, microinvasive and stage IB1 invasive neoplasia (Figures 3g-i). No difference is seen between these different stages of cancer progression (transformation zone/CIN3 

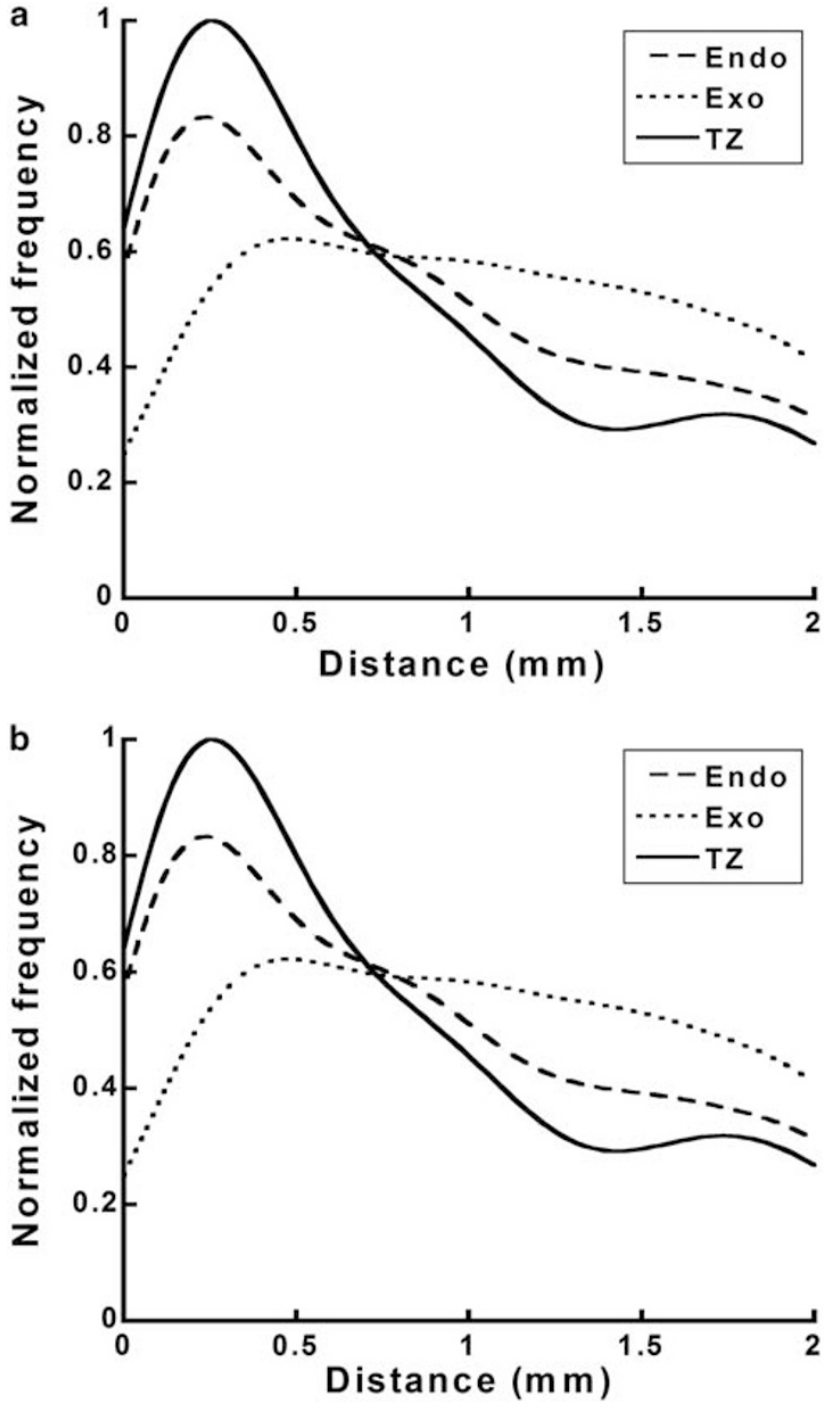

Figure 5 (a) Lymphatic vessel distribution under the three anatomical regions of the benign uterine cervix (Endo, endocervix; Exo, exocervix; TZ, transformation zone). (b) Lymphatic vessel distribution within the perineoplastic area of CIN3, microinvasive (MI) and 1B1 cervical lesions in comparison with non-pathological condition represented by the transformation zone of benign cervix (BC TZ). Curves were normalized taking the maximum of the control curve at one.

$P=0.0927, \quad$ transformation zone/microinvasive $P=0.5288$, transformation zone/invasive 1B1 cancers $P=0.4349$; Figure 3j). A further in depth quantification shows that a higher lymphatic vessel density is detected in the peritumoral area of IB1 cervical neoplasia compared with what was found in the 'deep tissue' of benign cervical tissue, stroma where lymphatic vessel density was initially low (superior to $2 \mathrm{~mm}$ from the adjacent epithelium; Supplementary Figure S1).

In order to specifically investigate the lymphangiogenic activity, proliferating lymphatic vessels were detected by double immunostaining using anti-D2/40 and anti-Ki67 antibodies (Figure 4a).
Table 1 Association between intra- and peritumoral LVD and clinicopathologic tumor feature (LVSI and lymph node status)

\begin{tabular}{|c|c|c|c|c|}
\hline & \multicolumn{2}{|c|}{ Intratumoral LVD } & \multicolumn{2}{|c|}{ Peritumoral LVD } \\
\hline & Mean \pm s.d. & $\mathrm{P}$-value & Mean \pm s.d. & $\mathrm{P}$-value \\
\hline \multicolumn{5}{|l|}{ Nodal metastasis } \\
\hline Absent $(n=34)$ & $8.57 \pm 7.47$ & 0.7214 & $9.52 \pm 4.12$ & 0.4455 \\
\hline Present $(n=12)$ & $7.78 \pm 3.79$ & & $9.33 \pm 6.03$ & \\
\hline \multicolumn{5}{|l|}{ LVSI } \\
\hline Absent $(n=21)$ & $7.10 \pm 7.80$ & 0.0550 & $8.76 \pm 4.64$ & 0.3777 \\
\hline Present $(n=25)$ & $9.21 \pm 5.62$ & & $10.72 \pm 6.27$ & \\
\hline
\end{tabular}

Abbreviations: LVD, lymphatic vessel density; LVSI, lymphovascular space invasion.

Results are represented as mean value \pm s.d. The Mann-Whitney test was performed for comparison.

The stromal density of proliferating lymphatic vessels is again higher under the transformation zone than under the exocervix $(P=0.0114$; Figure $4 \mathrm{~b})$. Transformation zone-associated proliferating lymphatic vessel density appears similar in benign cervix, CIN3 $(P=0.6189)$ and microinvasive lesions $(P=0.0889$; Figure 4c). Proliferating lymphatic vessel density appears reduced in stage IB1 cervical cancers compared with microinvasive lesions $(P<0.0001)$, CIN3 $(P=0.0081)$ and benign cervix $(P=0.0032)$.

\section{Lymphatic Vessel Distribution}

To better characterize the lymphatic vascular network, a global stromal analysis has been achieved by determining the distribution of lymphatic vessels in the region surrounding the epithelial layer in normal, preinvasive and invasive conditions. This generates vessel distribution curves allowing to determine the precise depth at which maximal vessel density is observed. In benign cervix, the distribution curve confirms the close vicinity between the lymphatic vessels and the epithelium basal membrane, both under the transformation zone and under the endocervix (Figure 5a). Indeed, the lymphatic vessel number peaks at a distance of $0.30 \mathrm{~mm} \pm 0.12$ of depth under transformation zone and $0.31 \mathrm{~mm} \pm 0.23$ under the endocervix, respectively. Oppositely, under the exocervix, the maximal vessel number is detected at $0.65 \mathrm{~mm} \pm 0.52$ to the epithelium, reaches a minimal value and remains constant thereafter. Notably, under the neoplastic epithelia, a shift of lymphatic vessel distribution toward the tumor edges is detected (Figure 5b). Indeed, the maximal lymphatic vessel density peaks at a lower distance under neoplasia compared with that found under the transformation zone of benign cervix (CIN3 $=0.13 \mathrm{~mm} \pm 0.07$, microinvasion $=0.13 \mathrm{~mm} \pm 0.08$ and IB1 cervical cancers $=$ $0.11 \mathrm{~mm} \pm 0.07$ versus transformation zone of benign 

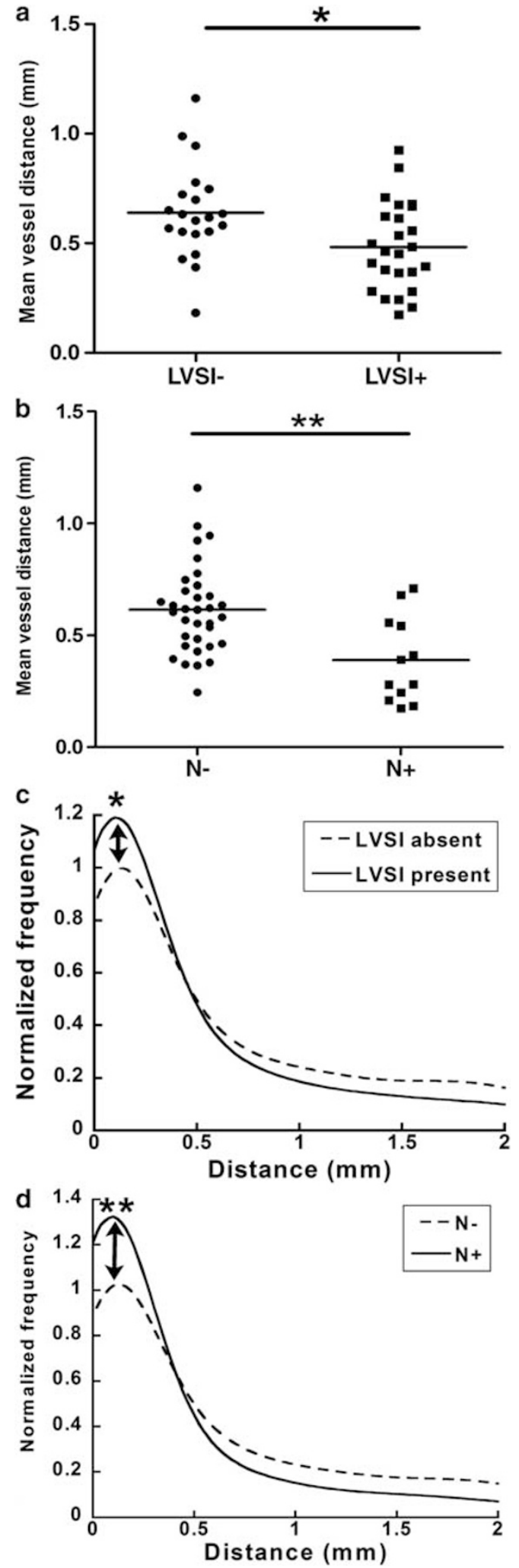

Figure $6(\mathbf{a}, \mathbf{b})$ Mean vessel distance between peritumoral lymphatic vessels and the tumor edges. (c, d) Lymphatic vessel distribution analysis within the peritumoral area of 1B1 cervical cancers. Comparisons were performed regarding the presence/ absence of lymphatic vascular space invasion (LVSI) and lymph node status $(\mathrm{N}+/ \mathrm{N}-)$. Curves were normalized taking the maximum of the control curve at one. ${ }^{*} P<0.05$, ${ }^{*} P<0.01$. cervix $=0.30 \pm 0.12 ; \quad P<0.05)$. Moreover, at this depth, a significant increase of relative vessel number (ranging from 60 to $89 \%$ ) is observed under the neoplastic lesions.

\section{Association between Lymphatic Vessel Density and Spatial Distribution with Lymph Node Status and Lymphovascular Space Invasion in IB1 Cervical Cancer}

We next focused our investigation on early stage cervical cancer patients (FIGO stage IB1) by exploring intra- and peritumoral lymphatic vasculature. Lymphatic vessel density and spatial lymphatic distribution were tested for their ability to predict lymphovascular space invasion and lymph node status. Intra- and peritumoral global lymphatic vessel density are neither associated with lymph node status nor with lymphovascular space invasion positivity (Table 1). Conversely, a smaller distance between the entire peritumoral lymphatic vessels and tumor edges is associated with those related lymphatic dissemination parameters (positive lymphovascular space invasion $=0.483 \mathrm{~mm} \pm 0.2$ versus negative lymphovascular space invasion $=0.640 \pm$ $0.21 \mathrm{~mm}, P=0.0162$ and positive lymph node $=$ $0.388 \pm 0.19$ versus negative lymph node $=0.66$ $\mathrm{mm} \pm 0.19, P=0.0039$; Figures $6 \mathrm{a}$ and $\mathrm{b}$ ). In fact, a detailed analysis of the lymphatic vessel spatial distribution reveals that while the number of peritumoral lymphatic vessels peaks at similar distance of the tumor edges (positive lymphovascular space invasion $=0.11 \mathrm{~mm} \pm 0.04$ negative lymphovascular space invasion $=$ versus $0.13 \mathrm{~mm} \pm 0.05 ; P>0.05$ and positive lymph node $=0.10 \pm 0.07$ versus negative lymph node $=0.013 \pm 0.04 ; P>0.05$ ), a higher number at this depth is correlated with lymphovascular space invasion $(P<0.05)$ and lymph node metastasis $(P<0.01$; Figures $6 c$ and $d)$.

\section{Discussion}

In early cervical cancer, the tumor lymphatic vasculature profile is thought to be useful to early predict the risk of lymph node extension. However, owing to several limitations associated to the conventional hot spot technique, no consensus currently exists. In the present work, a detailed analysis of the global cervical lymphatic vasculature has been performed using an original and reproducible quantitative computerized assisted method.

We describe for the first time a prominent lymphatic vessel density under the transformation zone of healthy cervical tissues, which has been characterized 30 years ago as the anatomical region where precancerous and cancerous lesions develop. ${ }^{26}$ Interestingly, no statistical difference between the transformation zone and the endocervix is due to the presence of immature metaplasia, zones that may be regarded as having a focal transformation 
zone effect in the endocervix. The transformation zone is increasingly drawing the attention of researchers. It results from a metaplastic conversion of glandular epithelial cells into squamous epithelium in response to a repetitive hormone induce vaginal $\mathrm{pH}$ acidification and sexual intercourse injury. ${ }^{33}$ This metaplastic process is often associated with local deregulation of cytokine and chemokine production as well as inflammatory response. Herfs et $a l^{34}$ hypothesized that this could lead to the establishment of an immunosuppressive microenvironment and potentiate tumor cell progression to the underlying stroma. Moreover, an elegant study reported that only a discrete population of cells at the squamocolumnar junction would be the precursors for most HPV-associated cervical carcinomas. ${ }^{27}$ In this context, the presence of a lymphangiogenic niche under the transformation zone of healthy cervical tissues reported herein suggests the existence of a specific microenvironment that could contribute to cancer progression. These data also argue that for using this region as a control to which neoplastic lymphatic vasculature has to be compared.

To date, an early lymphangiogenic switch during uterine cervical neoplasia progression appears well accepted in the literature. Schoppmann et $a l^{35}$ were first to observe a marked lymphatic vessel density under preinvasive lesions compared with normal cervical tissue but this failed to reach statistical significance. These results were then confirmed in three other studies that found a significant increase of lymphatic vessel density under CIN2/3 lesions or carcinoma in situ. ${ }^{10,11,15} \mathrm{~A}$ further rise of lymphangiogenesis in cervical cancers is supported by Longattho-Filho and Yang's studies ${ }^{10,14}$ while contradictory results were reported by Schoppmann et $a l^{35}$ and Gombos et $a{ }^{15}{ }^{15}$ Recently, Cimpean et al ${ }^{12}$ showed an activation of the lymphangiogenic activity in preinvasive and microinvasive before decreasing its intensity in early cervical cancers. However, in most studies, stromal region of benign cervical tissue that was considered as nonpathological condition (exocervix, endocervix or transformation zone) was either not mentioned ${ }^{10,35}$ or corresponded to the exocervix. ${ }^{12,15,36}$ Discrepancies between early increase of lymphatic vessel densities and our results showing a similar lymphatic vessel density detected under the transformation zone of benign cervix and preinvasive lesions are thought to be due to an inappropriate choice for the stromal region of benign cervix considered as control.

In our experience, during early steps of cancer progression (CIN3, micro-invasion and FIGO 1B1 neoplasia), no increase of lymphatic vessel density is found when compared with transformation zone of benign cervix. Our further observations of lymphatic vessel distribution indicate that, lymphatic vessel density maintains a stable level through the different stages of the neoplastic process secondary to lymphangiogenesis occurring in the deep tissue surrounding tumor invasive front in the stroma where lymphatic vessel density was initially low (superior to $2 \mathrm{~mm}$ from the adjacent epithelium). Moreover, the presence of Ki67-positive lymphatic vessels under any degrees of neoplasia supports that an ongoing lymphangiogenesis occurs during cancer progression. Interestingly, topographical modifications with a shift of lymphatic vessels toward the neoplastic edges are detected. Especially, despite similar lymphatic vessel density, a significant increase of the relative vessel number in the vicinity of neoplasia is found when compared the transformation zone of benign cervix. In accordance with the lymphangiogenic switch presented above, recent data reported high expression of VEGF-C and/or VEGF-D by neoplastic cells before the invasive process. ${ }^{9,11}$ In addition, Issa et $a l^{37}$ demonstrated that VEGF-C can promote tumor cell migration toward lymphatic vessels by increasing their proteolytic activity and motility as well as lymphatic secretion of the chemoattractive protein CCL21. Therefore, this supports the concept that lymphangiogenesis occurring during cancer progression is a continuum of the lymphangiogenic activity already present under the transformation zone of the benign cervix.

In patients with early cervical cancer, the lymphatic vessel density was proposed as a new promising factor capable to predict lymph node extension. Especially, most clinical studies showed that peritumoral lymphatic vessel density is associated with an aggressiveness behavior and appeared as a strong predictive factor of lymph node metastasis. ${ }^{14-21,36}$ However, contradictory results were reported by three others groups. ${ }^{10,23,24}$ Interestingly, a few years ago, Van der Auwera et al ${ }^{22}$ proposed that these discrepancies are caused by a lack of reproducibility when lymphatic vessel density is measured by the conventional optical microscopy (hot spot technique). They highlighted that reliable quantification technique needs to be developed for lymphangiogenesis quantification in human solid tumors. In the present work, we confirm that lymphatic vessel density determination through the hot spot technique is subjected to high intra- and inter-observer variability. On the contrary, the proposed computerized method shows high accuracy and reproducibility in lymphatic vessel detection. When global analysis of tumor lymphatic vasculature is performed, intra- and peritumoral lymphatic vessel density do not correlated neither to lymph node metastasis nor to lymphovascular space invasion. Nevertheless, we demonstrate that the distribution of the lymphatic vasculature must be taken into consideration when characterizing the lymphatic vessel density. Indeed, in IB1 cervical cancer, while similar lymphatic vessel density is found, an increase of the relative vessel number in the vicinity of the tumor edges is associated with lymphovascular space invasion and 
lymph node metastasis. This indicates that interactions between tumor and lymphatic endothelial cells leading to a modification of the spatial distribution of the lymphatic network could promote tumor cell dissemination. In this context, we aim to draw fundamental scientist's attention on the interest of a better characterization of the tumor and lymphatic endothelial cell interactions. A better understanding of such mechanisms could help to find new specific predictive maker of lymph node dissemination.

In conclusion, thanks to a novel computerassisted technique, a detailed reproducible and reliable quantification of the lymphatic vasculature can be performed on any immunostained tissue. This approach allows to overcome limitations associated with the hot spot technique and opens new perspectives in the field of pathology. Notably, we demonstrate that the spatial lymphatic vessel distribution has to be taken into account when the risk of nodal extension is tested. For these reasons, we argue that global analysis of the tumor vasculature is preferable to the traditional focal hot spot analysis. Moreover, such analysis highlights the need to concentrate on a better understanding of tumor cell and lymphatic vessel interactions that are involved in topographical modifications of the lymphatic vasculature during cancer progression. Of great interest is our finding of a specific lymphangiogenic niche under the transformation zone where cell transformation occurs. Finally, similarly to cervical cancer, prognostic value of the tumor lymphatic vessel density has been studied in other human solid tumors such as breast, colorectal, prostate, non-small cell lung cancers and melanoma and interestingly contradictory results were frequently observed. ${ }^{38}$ Consequently, the technology developed in this study could potentially be used to better characterize the lymphatic vasculature and the risk of nodal extension in the context of other tumors.

\section{Acknowledgments}

We address a particular acknowledgment to Paulette Herlin from Groupe de recherche en informatique, image, automatique et instrumentation of Caen (GREYC, University of Caen, France) for her technical support for image decimation. This work was supported by grants from the FP7-HEALTH-2007-A proposal no. 201279 'MICROENVIMET', the Fonds de la Recherche Scientifique Médicale, the Fonds de la Recherche Scientifique-FNRS (FRS-FNRS, Belgium), the Foundation against Cancer (foundation of public interest, Belgium), the CGRI-FNRSINSERM Coopération, the Fonds spéciaux de la Recherche (University of Liège), the Centre Anticancéreux près l'Université de Liège, the Fonds Léon Fredericq (University of Liège), the Direction Générale Opérationnelle de l'Economie, de l'Emploi et de la Recherche from the SPW (Région Wallonne, Belgium), the Fonds Social Européen (FSE, Belgium), the Fonds d'Investissements de la Recherche Scientifique (FIRS, CHU, Liège, Belgium), the Interuniversity Attraction Poles Programme-Belgian Science Policy (Brussels, Belgium), the Plan National Cancer (Service Public Fédéral). The Actions de Recherche Concertées (University of Liege, Belgium). CB is recipient of a Télévie-FNRS grant.

\section{Disclosure/conflict of interest}

The authors declare no conflict of interest.

\section{References}

1 Leong SP, Zuber M, Ferris RL, et al. Impact of nodal status and tumor burden in sentinel lymph nodes on the clinical outcomes of cancer patients. J Surg Oncol 2011;103:518-530.

2 Delgado G, Bundy B, Zaino R, et al. Prospective surgical-pathological study of disease-free interval in patients with stage IB squamous cell carcinoma of the cervix: a Gynecologic Oncology Group study. Gynecol Oncol 1990;38:352-357.

3 Hacker NF, Wain GV, Nicklin JL. Resection of bulky positive lymph nodes in patients with cervical carcinoma. Int J Gynecol Cancer 1995;5:250-256.

4 Sakuragi N. Up-to-date management of lymph node metastasis and the role of tailored lymphadenectomy in cervical cancer. Int J Clin Oncol 2007;12:165-175.

5 Benedetti-Panici P, Maneschi F, Scambia G, et al. Lymphatic spread of cervical cancer: an anatomical and pathological study based on 225 radical hysterectomies with systematic pelvic and aortic lymphadenectomy. Gynecol Oncol 1996;62:19-24.

6 Tammela T, Alitalo K. Lymphangiogenesis: molecular mechanisms and future promise. Cell 2010;140:460-476.

7 Sevin BU, Lu Y, Bloch DA, et al. Surgically defined prognostic parameters in patients with early cervical carcinoma. A multivariate survival tree analysis. Cancer 1996;78:1438-1446.

8 Ji RC. Lymphatic endothelial cells, tumor lymphangiogenesis and metastasis: new insights into intratumoral and peritumoral lymphatics. Cancer Metastasis Rev 2006;25:677-694.

9 Van Trappen PO, Steele D, Lowe DG, et al. Expression of vascular endothelial growth factor (VEGF)-C and VEGF-D, and their receptor VEGFR-3, during different stages of cervical carcinogenesis. J Pathol 2003;201: 544-554.

10 Longatto-Filho A, Pinheiro C, Pereira SM, et al. Lymphatic vessel density and epithelial D2-40 immunoreactivity in pre-invasive and invasive lesions of the uterine cervix. Gynecol Oncol 2007;107:45-51.

11 Jach R, Dulinska-Litewka J, Laidler P, et al. Expression of VEGF, VEGF-C and VEGFR-2 in in situ and invasive SCC of cervix. Front Biosci (Elite Ed) 2010;2:411-423.

12 Cimpean AM, Mazuru V, Cernii A, et al. Detection of early lymphangiogenesis by lymphatic microvascular density and endothelial proliferation status in preneoplastic and neoplastic lesions of the uterine cervix. Pathol Int 2011;61:395-400. 
13 Utrera-Barillas D, Castro-Manrreza M, Castellanos E, et al. The role of macrophages and mast cells in lymphangiogenesis and angiogenesis in cervical carcinogenesis. Exp Mol Pathol 2010;89:190-196.

14 Yang S, Cheng H, Cai J, et al. PlGF expression in preinvasive and invasive lesions of uterine cervix is associated with angiogenesis and lymphangiogenesis. APMIS 2009;117:831-838.

15 Gombos Z, Xu X, Chu CS, et al. Peritumoral lymphatic vessel density and vascular endothelial growth factor $\mathrm{C}$ expression in early-stage squamous cell carcinoma of the uterine cervix. Clin Cancer Res 2005;11: 8364-8371.

16 Gao P, Zhou GY, Yin G, et al. Lymphatic vessel density as a prognostic indicator for patients with stage I cervical carcinoma. Hum Pathol 2006;37:719-725.

17 Zhang SQ, Yu H, Zhang LL. Clinical implications of increased lymph vessel density in the lymphatic metastasis of early-stage invasive cervical carcinoma: a clinical immunohistochemical method study. BMC Cancer 2009;9:64.

$18 \mathrm{Yu} \mathrm{H}$, Zhang S, Zhang R, et al. The role of VEGF-C/D and Flt-4 in the lymphatic metastasis of early-stage invasive cervical carcinoma. J Exp Clin Cancer Res 2009;28:98.

19 Saad RS, Ismiil N, Ghorab Z, et al. Lymphatic vessel density as a prognostic marker in clinical stage I endocervical adenocarcinoma. Int J Gynecol Pathol 2010;29:386-393.

$20 \mathrm{Liu} \mathrm{H}$, Xiao J, Yang Y, et al. COX-2 expression is correlated with VEGF-C, lymphangiogenesis and lymph node metastasis in human cervical cancer. Microvasc Res 2011;82:131-140.

21 Cai L, Yang S, Ding $\mathrm{H}$, et al. Tumor-associated lymphatic endothelial cell promotes invasion of cervical cancer cells. APMIS 2013 (e-pub ahead of print).

22 Van der Auwera I, Cao Y, Tille JC, et al. First international consensus on the methodology of lymphangiogenesis quantification in solid human tumours. Br J Cancer 2006;95:1611-1625.

23 Sotiropoulou N, Bravou V, Kounelis S, et al. Tumour expression of lymphangiogenic growth factors but not lymphatic vessel density is implicated in human cervical cancer progression. Pathology 2010;42:629636.

24 Xiong Y, Cao LP, Rao HL, et al. Clinical significance of peritumoral lymphatic vessel density and lymphatic vessel invasion detected by D2-40 immunostaining in FIGO Ib1-IIa squamous cell cervical cancer. Cell Tissue Res 2012;348:515-522.
25 Balsat C, Blacher S, Signolle N, et al. Whole slide quantification of stromal lymphatic vessel distribution and peritumoral lymphatic vessel density in early invasive cervical cancer: a method description. ISRN Obstet Gynecol 2011;2011:354861.

26 Burghardt E, Ostor AG. Site and origin of squamous cervical cancer: a histomorphologic study. Obstet Gynecol 1983;62:117-127.

27 Herfs M, Yamamoto Y, Laury A, et al. A discrete population of squamocolumnar junction cells implicated in the pathogenesis of cervical cancer. Proc Natl Acad Sci U S A 2012;109:10516-10521.

28 Lester SC. Manual of Surgical Pathology, Vol. Churchill Livingstone 2001.

29 Mukonoweshuro P, Oriowolo A, Smith M. Audit of the histological definition of cervical transformation zone. J Clin Pathol 2005;58:671.

30 Tsai WH. Moment-preserving thresholding: a new approach. Computer Vision, Graphics Image Processing 1995;29:377-393.

31 Françoise R MJJ, Plancoulaine B, Herlin P. Optimal resolution for automatic quantification of blood vessels on digitized images of the whole cancer section. Image Analysis Stereol 2005;24:59-67.

32 Weidner N, Semple JP, Welch WR, et al. Tumor angiogenesis and metastasis-correlation in invasive breast carcinoma. N Engl J Med 1991;324:1-8.

33 Delvenne P, Herman L, Kholod N, et al. Role of hormone cofactors in the human papillomavirusinduced carcinogenesis of the uterine cervix. Mol Cell Endocrinol 2007;264:1-5.

34 Herfs M, Hubert P, Delvenne P. Epithelial metaplasia: adult stem cell reprogramming and (pre)neoplastic transformation mediated by inflammation? Trends Mol Med 2009;15:245-253.

35 Schoppmann SF, Schindl M, Breiteneder-Geleff S, et al. Inflammatory stromal reaction correlates with lymphatic microvessel density in early-stage cervival cancer. Anticancer Res 2001;21:3419-3423.

36 En-Lin S, Wei-Wei Y, Xiao-Liang X, et al. Relationship between high density of peritumoral lymphatic vessels and biological behavior of cervical cancer. Int J Gynecol Cancer 2012;22:1435-1441.

37 Issa A, Le TX, Shoushtari AN, et al. Vascular endothelial growth factor-C and C-C chemokine receptor 7 in tumor cell-lymphatic cross-talk promote invasive phenotype. Cancer Res 2009;69:349-357.

38 Sleeman JP, Thiele W. Tumor metastasis and the lymphatic vasculature. Int J Cancer 2009;125: $2747-2756$.

Supplementary Information accompanies the paper on Modern Pathology website (http://www.nature.com/ modpathol) 